\section{Stotternd Deutsch lernen}

Stottern ist eine unfreiwillige Störung im Redefluss, die Männer etwa viermal häufiger betrifft als Frauen und meist bereits im Kindesalter auftritt. Etwa $1 \%$ der Bevölkerung stottert. Die Kernsymptome sind Wiederholungen, Dehnungen und Blockaden [1].

\section{Ursache und Remission}

Neef et al. fanden mithilfe von MRT heraus, dass zu einer hohen Wahrscheinlichkeit rechtshemisphärisch in der unteren Windung des Frontalhirns zu viel Aktivität vorherrscht, sodass es zu einer übermäßigen Hemmung kommt. Diese kann ein plötzliches Stoppen jeglicher Bewegungsabläufe auslösen. Damit wird verifiziert, was bereits andere Studien aufweisen, nämlich dass bei Stotternden ein Ungleichgewicht der Hirnaktivität beider Hirnhälften besteht [2].

Die Remission des Stotterns kann bis zum 13. Lebensjahr mit einer Wahrscheinlichkeit von $75 \%$ gelingen [3]. Etwa ab dem 14. Lebensjahr ist eine Remission sehr unwahrscheinlich. Betroffene lernen daher, ihr Stottern zu akzeptieren und zu modifizieren.

\section{Therapie}

Innerhalb der Stottertherapie wird in zwei Therapieströmungen unterschieden: Stottermodifikation und Fluency Shaping. Die Stottermodifikation verläuft in vier Phasen:
- der Identifikation eigener und fremder Stottersymptome,

- der Desensibilisierung gegenüber den negativen Gefühlen bezüglich des Stotterns,

- der Modifikation,

- der Stabilisierung.

Fluency Shaping besteht im Vergleich dazu aus einer Modifikation des gesamten Sprechens. Die Begleiterscheinungen des Stotterns wie Sprechangst werden nicht direkt behandelt, sondern Stotternde erarbeiten eine neue Sprechweise, die anstelle des Stotterns eingesetzt werden kann [4]. Als Beispiel eines kombinierten Vorgehens weist der Ansatz der Bonner Stottertherapie einen signifikanten Therapieeffekt auf, der sich in einem offenen Umgang Stotternder mit ihrem eigenen Stottern zeigt [5].

\section{Einer von Deutschlands 820000 Stotternden}

Ein 24 Jahre alter Mathematik-Student aus Togo mit französischer Muttersprache lebt seit dem Frühjahr 2016 in Deutschland. Er besucht einen Sprachkurs, da er in Deutschland ein Informatikstudium beginnen möchte. Durch die mündliche Abschlussprüfung des Deutschkurses fällt er nicht etwa deshalb, weil er mangelnde Deutschkenntnisse aufweist, sondern weil er stottert. Er begibt sich in Behandlung und spricht innerhalb von 5 Monaten so flüssig, dass er im Gespräch gut verstanden wird.

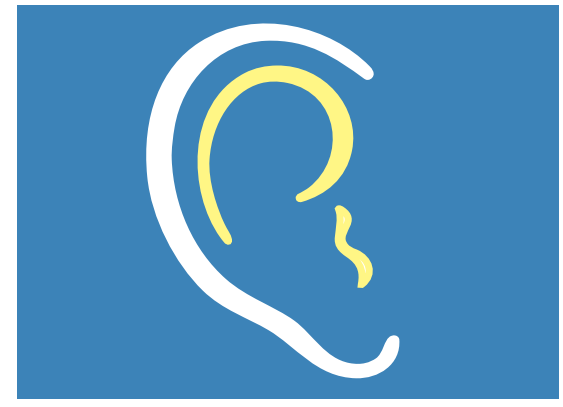

Der Patient zeigt (Tonbeispiel 1) ein schweres originäres neurogenes nichtsyndromales Stottern [4] mit häufigen Blockaden, seltenen Dehnungen sowie Wiederholungen. Die besondere Auffälligkeit seines Stotterns ist gekennzeichnet durch verbale Begleitsymptome in Form von Wort- und Satzteilwiederholungen, die ihm als Aufschub dienen. In dem Moment, in dem er sein Stottern wahrnimmt, benutzt er statt des gefürchteten Wortes ein anderes, das schon vorher flüssig gesprochen werden konnte. Wie das Tonbeispiel 1 zeigt, kostet ihn dies nicht nur viel Konzentration, sondern auch Zeit. Innerhalb der logopädischen Therapie lernt er, die Begleitsymptome zu unterbinden, sodass nur Kernsymptome im Sprechen aufkommen (Tonbeispiel 2).

Er durchläuft die Phasen der Identifikation und Desensibilisierung zügig. Im Therapieverlauf zeigt sich, dass das primäre Stottern lediglich in leichter Ausprägung besteht. Somit entscheidet sich der Patient dafür, keine Techniken einzusetzen, sondern sein gelegentlich auftretendes Stottern offen zu zeigen.
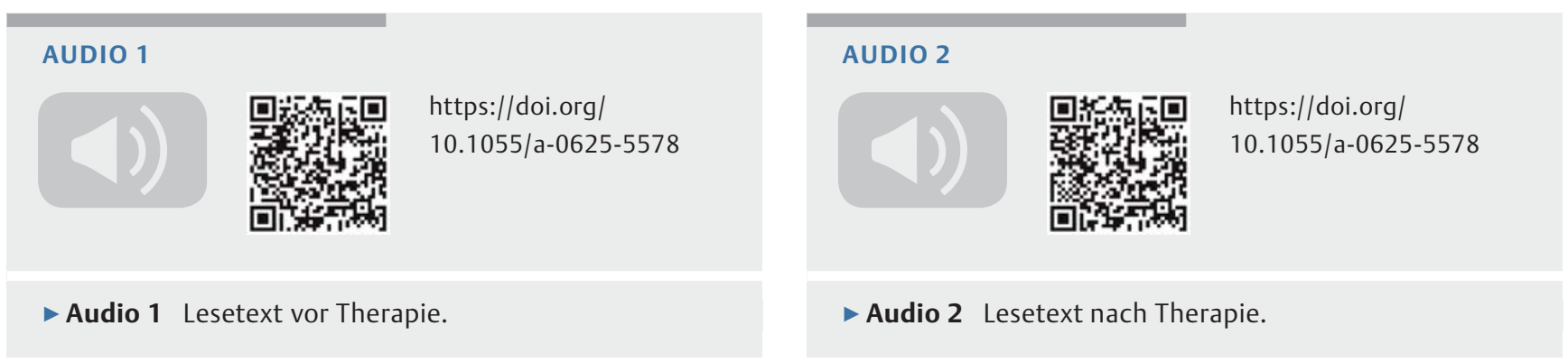
Bei einem zufälligen Treffen der Therapeutin und dem Patienten im Linienbus kommt es zu einer Unterhaltung über sein Informatikstudium. Er spricht trotz einer großen Zuhörerzahl und Hintergrundgeräuschen sehr offen, laut und sichtlich entspannt. Eine Dame lauscht vorerst und nimmt schließlich am Gespräch teil. Als der Patient aus dem Bus steigt, sagt die Dame zur Therapeutin: „Hier sieht man, wie gut Integration gelingen kann. Wie sympathisch dieser junge Mann ist und wie schlau! “ Dass er stottert, hatte sie nicht bemerkt.

FAZIT

Auch wenn der betroffene Patient sein Stottern nie ganz verliert, konnte die Therapie erfolgreich abgeschlossen werden. Laut ICF-Konzept erzielen Stottertherapien eine Reduktion der Anzahl und des Kraftaufwandes von stottertypischen Unflüssigkeiten sowie einen Abbau der Begleitsymptome (vgl. [4]). Eine Stottertherapie ist somit immer dann gelungen, wenn die gemeinsam vereinbarten Therapieziele erreicht wurden. Das Ziel dieses Patienten war ein „flüssigeres und angstfreies Sprechen“.

Wencke Röhrenbeck, M. A., Mainz
Literatur

[1] Sandrieser P, Schneider P, Springer L et al. Hrsg. Thiemes Forum Logopädie: Stottern im Kindesalter. Stuttgart, New York. Thieme 2008

[2] Neef N, Anwander A, Bütfering C et al. Structural connectivity of right frontal hyperactive areas scales with stuttering severity. Brain 2018; 141: 191-204

[3] Wendlandt W, Springer L, Schrey-Dern D. Hrsg. Thiemes Forum Logopädie: Stottern im Erwachsenenalter. Stuttgart, New York. Thieme 2009

[4] Neumann K, Euler H., Bosshardt HG et al. Hrsg. Deutsche Gesellschaft für Phoniatrie und Pädaudiologie. Pathogenese, Diagnostik und Behandlung von Redeflussstörungen. Evidenz- und konsensbasierte S3-Leitlinie, AWMF-Registernummer 049-013, Version 1. 2016

[5] Ude GE, Prüß H, Richardt K et al. Die Angst vor dem Sprechen - eine Untersuchung zur Wirksamkeit des Angstabbaus im Rahmen der Bonner Stottertherapie. Forschung Sprache $2016 \mathrm{Jg}$. 04; 02: 20-35 